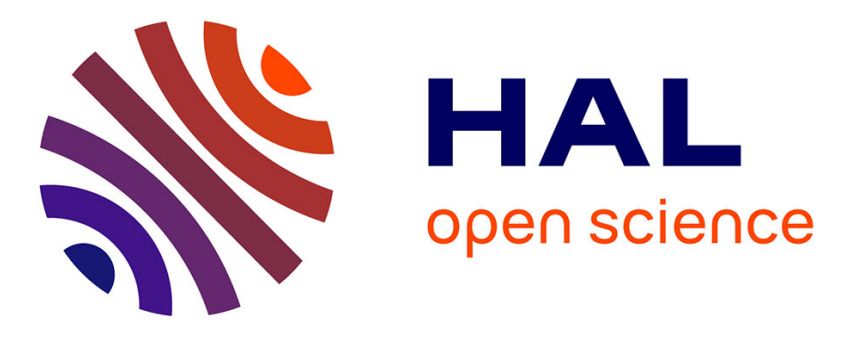

\title{
Asynchronous Inter-Level Forward-Checking for DisCSPs
}

\author{
Redouane Ezzahir, Christian Bessiere, Mohamed Wahbi, Imade Benelallam, \\ El Houssine Bouyakhf
}

\section{- To cite this version:}

Redouane Ezzahir, Christian Bessiere, Mohamed Wahbi, Imade Benelallam, El Houssine Bouyakhf. Asynchronous Inter-Level Forward-Checking for DisCSPs. CP: Principles and Practice of Constraint Programming, Sep 2009, Lisbon, Portugal. pp.304-318, 10.1007/978-3-642-04244-7_25 . lirmm00545541

\section{HAL Id: lirmm-00545541 https://hal-lirmm.ccsd.cnrs.fr/lirmm-00545541}

Submitted on 9 Oct 2018

HAL is a multi-disciplinary open access archive for the deposit and dissemination of scientific research documents, whether they are published or not. The documents may come from teaching and research institutions in France or abroad, or from public or private research centers.
L'archive ouverte pluridisciplinaire HAL, est destinée au dépôt et à la diffusion de documents scientifiques de niveau recherche, publiés ou non, émanant des établissements d'enseignement et de recherche français ou étrangers, des laboratoires publics ou privés. 


\title{
Asynchronous Inter-Level Forward-Checking for DisCSPs
}

\author{
Redouane Ezzahir ${ }^{1,2}$, Christian Bessiere ${ }^{1}$, Mohamed Wahbi $^{1,2}$, Imade Benelallam ${ }^{2}$, \\ and El Houssine Bouyakhf ${ }^{2}$ \\ 1 LIRMM/CNRS, U. of Montpellier 2, France \\ bessiere@lirmm.fr, ezzahir@lirmm.fr,wahbi@lirmm.fr \\ ${ }^{2}$ LIMIARF/FSR, U. of Mohammed V Agdal, Morroco \\ imade.benelallam@ieee.org, bouyakhf@fsr.ac.ma
}

\begin{abstract}
We propose two new asynchronous algorithms for solving Distributed Constraint Satisfaction Problems (DisCSPs). The first algorithm, AFC-ng, is a nogood-based version of Asynchronous Forward Checking (AFC). The second algorithm, Asynchronous Inter-Level Forward-Checking (AILFC), is based on the AFC-ng algorithm and is performed on a pseudo-tree ordering of the constraint graph. AFC-ng and AILFC only need polynomial space. We compare the performance of these algorithms with other DisCSP algorithms on random DisCSPs in two kinds of communication environments: Fast communication and slow communication. Our experiments show that AFC-ng improves on AFC and that AILFC outperforms all compared algorithms in communication load.
\end{abstract}

\section{Introduction}

Distributed Constraint Satisfaction Problems (DisCSPs) is a general framework for solving distributed problems. DisCSPs have a wide range of applications in multi-agent coordination, such as distributed resource allocation problems [1], distributed scheduling problems [2], sensor networks [3], and log-based reconciliation [4].

DisCSPs are composed of agents, each holding its local constraint network. Variables in different agents are connected by constraints. Agents assign values to their variables, attempting to generate a locally consistent assignment that is also consistent with all constraints between agents [5,6]. To achieve this goal, agents check the value assignments to their variables for local consistency and exchange messages among them to check consistency of their proposed assignments against constraints among variables that belong to different agents.

Several efficient distributed algorithms for solving DisCSPs have been developed in the last decade. Synchronous Backtrack (SBT) is the simplest DisCSP search algorithm that performs assignments sequentially and synchronously. Only the agent holding a Current Partial Assignment (CPA) performs an assignment or backtrack [7]. The first complete asynchronous search algorithm for DisCSPs is the Asynchronous Backtracking $\mathrm{ABT}[8,5,9]$. In $\mathrm{ABT}$, agents perform assignments asynchronously and send out messages to constraining agents, informing them about their assignments. Due to the asynchronous nature of agents operations, the global assignment state at any particular time during the run of asynchronous backtracking is in general inconsistent. Nogoods 
are used to prevent the construction of globally inconsistent solutions. Another promising algorithm for DisCSPs is the Asynchronous Forward-Checking (AFC) algorithm $[10,11]$. This algorithm is based on the forward checking (FC) algorithm for CSPs, but performs forward checking asynchronously.

In this paper, we present two new asynchronous algorithms for solving DisCSPs. The first one is based on Asynchronous Forward Checking (AFC) and uses nogood recording. We call it Nogood-Based AFC ( AFC-ng). The second one is based on AFCng and is performed on a pseudo-tree ordering of the constraint graph. We call it Asynchronous Inter-Level Forward-Checking (AILFC).

This paper is organized as follows. Section 2 gives the necessary background on DisCSPs. Sections 3 and 4 describe the algorithms AFC-ng and AILFC. Correctness proofs are given in Section 5. Section 6 presents an experimental evaluation of our proposed algorithms against three other well-known distributed algorithms. Section 7 summarizes several related works and we conclude the paper in Section 8.

\section{Background}

\subsection{Distributed Constraint Satisfaction Problems}

The Distributed Constraint Satisfaction Problem DisCSP has been formalized in [6] as a tuple $(\mathcal{A}, \mathcal{X}, \mathcal{D}, \mathcal{C})$, where $\mathcal{A}$ is a set of agents $\left\{A_{1}, \ldots, A_{k}\right\}, \mathcal{X}$ is a set of variables $\left\{x_{1}, \ldots, x_{n}\right\}$, where each $x_{i}$ is controlled by one agent in $\mathcal{A} . \mathcal{D}=\left\{D\left(x_{1}\right), \ldots, D\left(x_{n}\right)\right\}$ is a set of domains, where $D\left(x_{i}\right)$ is a finite set of values to which variable $x_{i}$ may be assigned. Only the agent who is assigned a variable has control on its value and knowledge of its domain. $\mathcal{C}$ is a set of binary constraints that specify the combinations of values allowed for the two variables they involve. A constraint $c_{i j} \in \mathcal{C}$ between two variables $x_{i}$ and $x_{j}$ is a subset of the Cartesian product $D\left(x_{i}\right) \times D\left(x_{j}\right)$.

For simplicity purposes, we consider a restricted version of DisCSP where each agent owns exactly one variable. We identify the agent number with its variable index. We also consider that the total order among agents that is used by a search algorithm is the lexicographic ordering $x_{i} \prec x_{j}$ if $i<j$.

We assume that communication between two agents is not necessarily generalized FIFO (aka causal order) channels [12]. Thus, all agents maintain their own counter, called $C t r$, and increment it whenever they change their value. The current value of the counter tags each generated assignment. An assignment for an agent $A_{i} \in \mathcal{A}$ is a tuple $\left(x_{i}, v_{i}, C t r_{i}\right)$ where $v_{i}$ is a value from the domain of $x_{i}$ and $C t r_{i}$ is the tag value.

A nogood $n g$ for value $c$ for variable $x_{k}$ is a clause of the form $x_{i}=a \wedge x_{j}=$ $b \wedge \ldots \Rightarrow x_{k} \neq c$, meaning that the assignment $x_{k}=c$ (i.e., the right hand side $R h s(n g)$ of $n g)$ is inconsistent with the assignments $x_{i}=a, x_{j}=b, \ldots$ (i.e., the left hand side $L h s(n g)$ of $n g$ ). When every value of a variable $x_{k}$ is ruled out by a nogood, these nogoods are resolved computing a new nogood new $N g$. Let $x_{j}$ be the lowest variable in the left-hand side of the nogoods, with $x_{j}=b$. Lhs $($ new $N g)$ is the conjunction of the left-hand sides of all nogoods except $x_{j}=b . R h s($ new $N g)$ is $x_{j} \neq b$. 
Definition 1 (Current Partial Assignment (CPA)). Given an agent $A_{i} \in \mathcal{A}$, a CPA is an ordered set of assignments $\left\{\left(x_{1}, v_{1}, C t r_{1}\right), \ldots,\left(x_{i-1}, v_{i-1}, C t r_{i-1}\right) \mid x_{1} \prec \ldots \prec\right.$ $\left.x_{i-1} \prec x_{i}\right\}$.

Definition 2 (AgentView). The agent view of an agent $A_{i} \in \mathcal{A}$ stores the newest assignments received from agents that precede $A_{i}$ in the ordering $\prec$. It has a form similar to a CPA and is initialized to the set of empty assignments $\left\{\left(x_{j}, \emptyset, 0\right) \mid i \neq j\right\}$.

Definition 3 (Time-stamp). A time-stamp is an ordered list of counters $\left\langle C t r_{1}, \mathrm{Ctr}_{2}\right.$, $\left.\ldots, C t r_{k}\right\rangle$. When comparing (lexicographically) two time-stamps, the most up to date is one which is lexicographically greater, that is, the one with greatest value on the first counter on which they differ, if any, otherwise the longest one.

\subsection{Asynchronous Forward-Checking (AFC)}

AFC is based on the Forward-Checking (FC) algorithm for CSPs but it performs the forward checking phase asynchronously $[10,11]$. As in synchronous backtracking, agents assign their variables only when they hold the current partial assignment (CPA). The CPA is a unique message that is passed from one agent to the next one in the ordering. The CPA carries the partial assignment that agents attempt to extend into a complete solution by assigning their variables on it. Forward checking is performed as follows. Every agent that sends the CPA to its successor also sends copies of the CPA to all agents whose assignments are not yet on the CPA. Agents that received CPAs update domains of their variables, removing all values that are in conflict with assignments on the received CPA.

An agent that generates an empty domain as a result of a forward-checking operation initiates a backtrack by sending Not_OK messages which carry the inconsistent partial assignment which caused the empty domain. Not_OK messages are sent to all agents with unassigned variables on the (inconsistent) CPA. When an agent holding a Not_OK receives a CPA, it sends this CPA back in a backtrack message. When multiple agents reject a given assignment by sending Not_OK messages, only the first agent that will receive a CPA and is holding a relevant Not_OK message will eventually backtrack. After receiving a new CPA, the Not_OK message becomes obsolete when the CPA it carries is no longer a subset of the received CPA.

An improved backtrack method for AFC was described in Section 6 of [11]. Instead of just sending Not_OK messages to all agents unassigned in the CPA, the agent who detects the empty domain can itself initiate a backtrack operation. It sends a backtrack message to the last agent assigned in the inconsistent CPA in addition to the Not_OK messages to all agents not instantiated in the inconsistent CPA. The agent who receives a backtrack message generates (if it is possible) a new CPA that will dominate older ones thanks to the time-stamp mechanism (see Definition 3).

\section{Nogood-based AFC}

The nogood-based Asynchronous Forward-Checking (AFC-ng) is based on AFC but it tries to enhance the asynchronism of the forward phase. The two main features of 


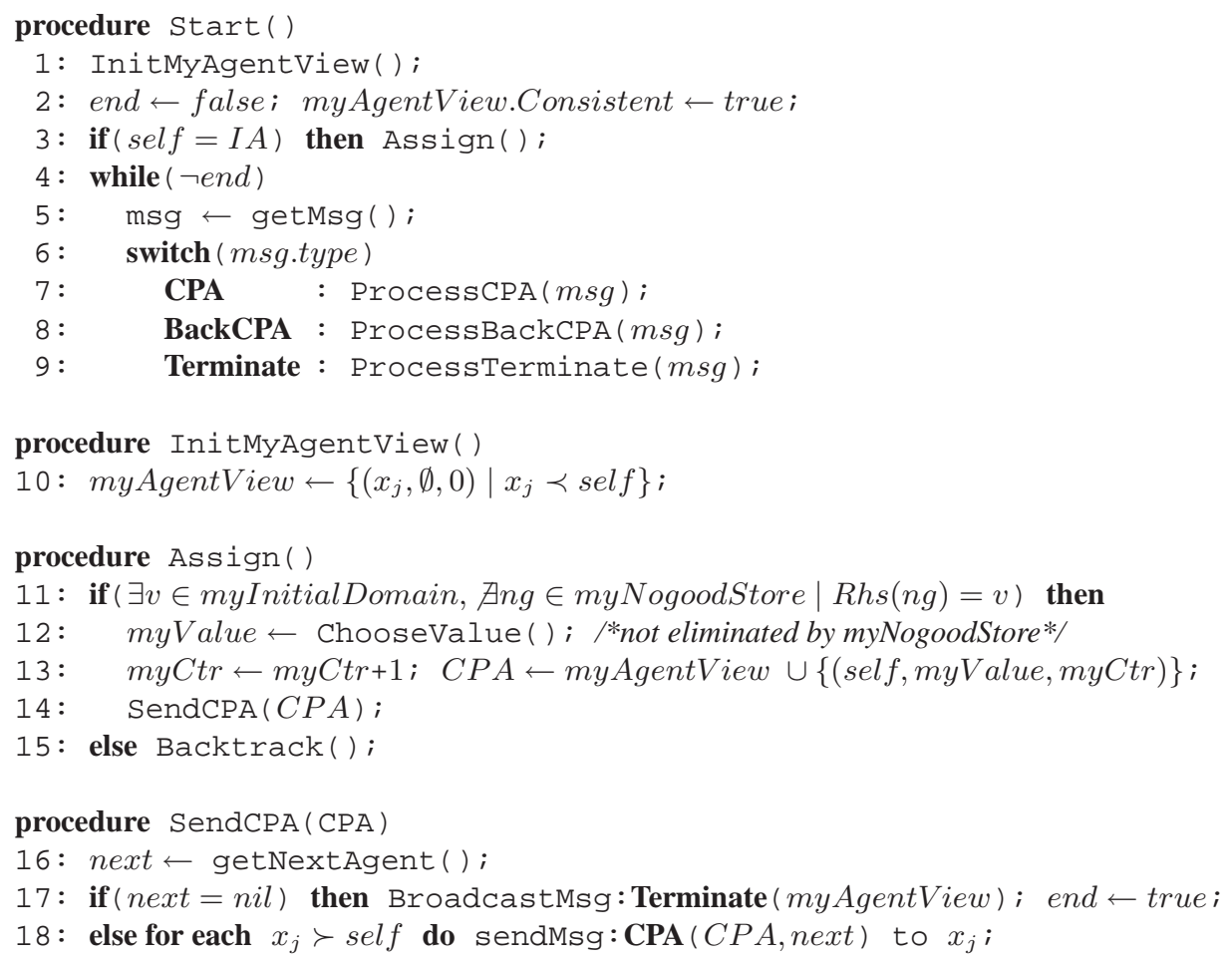

Fig. 1. Nogood-based AFC algorithm running by agent self (Part 1)

AFC-ng are the following. First, an agent finding an empty domain no longer sends Not_OK messages. It resolves the nogoods attached to its values and sends the backtrack message to the lower agent in the resolved nogood. Hence, multiple backtracks may be performed at the same time coming from different agents having an empty domain. These backtracks are sent concurrently by these different agents to different destinations. The re-assignments of the destination agents then happen simultaneously and generate several CPAs. However, the CPA coming from the highest level in the search tree will eventually dominate all others. Interestingly, the search process with the new CPA of highest level can use nogoods reported by the (killed) lower level processes, so that it benefits from their computational effort. Second, each time an agent performs a forward-check, it revises its initial domain, (including values already removed by a stored nogood) in order to store the best nogoods for removed values (one nogood per value). When comparing two nogoods eliminating the same value, the nogood with the highest possible lowest variable involved is selected (HPLV heuristic) [13]. As a result, when an empty domain is found, the resolvent nogood contains variables as high as possible in the ordering, so that the backtrack message is sent as high as possible, thus saving unnecessary search effort [9].

\section{Description of the algorithm}

We call sel $f$ the variable that points to the agent itself. An AFC-ng agent sel $f$ executes 
the code shown in Figures 1 and 2. The data structure. myInitialDomain contains all values of the initial domain of self. self stores a nogood per removed value in myNogoodStore. self calls the procedure Start() in which self initiates its agent view (line 1) by setting counters to zeros (line 10). The agent view contains a consistency flag that represents whether the partial assignment it holds is consistent. If self is the initializing agent $(I A)$, it initiates the search by calling procedure assign() (line 3$)$. All agents performing the main loop wait for messages, and process received messages according to their types (line 4-9).

When calling assign() self tries to find an assignment, which is consistent with its agent view. If self succeeds, it increments its counter $C t r$, generates a CPA from its agent view augmented by sel $f$ assignment (line 13), and then sends forward the CPA to every agent whose assignments are not yet on the CPA, or reports a solution, when the CPA includes all agents assignments (line 17). Before sending any CPA, self attaches to every CPA message the ID of his successor (line 18). Only if the receiver ID equals that attached to the CPA message, the receiver performs an assignment (line 26). When self fails to find a consistent assignment, it calls procedure Backtrack() (line 15).

Agents use time-stamps to detect and discard obsolete CPAs. Function CompareTimeStamp(view, CPA) returns the index splitlevel of the first counter on which view and CPA differ if CPA is newest (see Definition 3) or contains view (line 48). If view is newest, it returns -1. When view and CPA are identical or when CPA is included in view CompareTimeStamp returns 0.

Whenever self receives a CPA, procedure ProcessCPA() is called. self $f$ checks its agent view status. If it is not consistent and the agent view is a subset of the received CPA, this means that self has already backtracked, then self does nothing (line 19). Otherwise, self compares the time-stamp of its agent view with the one of the received CPA by calling CompareTimeStamp (line 20). If the received CPA is newest, self updates its agent view and marks it consistent (lines 21-22). Procedure UpdateMyAgentView (lines 41-43) sets the agent view and the nogood store to be consistent with the received CPA. Each nogood in the nogood store containing a value for a variable different from that received in the CPA will be deleted (line 43). Next, self calls procedure FC_ReviseInitialDomain() (in line 23) to store nogoods for values inconsistent with the new agent view or to try to find a better nogood for values already having one in the nogood store (line 46). A nogood is better according to the HPLV heuristic if the lowest variable in the body of the nogood is higher.

When every value of self's variable is ruled out by a nogood (line 24), the procedure Backtrack is called. These nogoods are resolved by computing a new nogood new $N g$ (line 27). If the new nogood is empty, self terminates execution after sending a Terminate message to all agents in the system meaning that problem is unsolvable (line 28). Otherwise, self updates its agent view by removing assignments of every agent that is strictly greater than the last agent $(R h s($ new $N g))$ in the new $N g$. self also updates its nogood store by removing obsolete nogoods. Finally it marks its agent view as inconsistent and it initiates a backtrack procedure by sending one BackCPA message to the lower priority agent $(R h s(n e w N g)$ ) involved in the new $N g$ (line 34).

The BackCPA message carries the new $N g$ and the inconsistent CPA containing assignments of all agents smaller than or equal to Rhs $(n e w N g)$ in the agent ordering 
ProcessCPA $(\mathrm{msg})$

19: if ( $\neg$ my AgentView.Consistent $\wedge$ myAgentView $\subset$ msg.CPA) then return;

20: splitlevel $\leftarrow$ CompareTimeStamp (myAgentView, msg.CPA);

21: if (splitlevel $>0$ ) then

22: UpdateMyAgentView (msg.CPA, splitlevel) ; myAgentView.Consistent $\leftarrow$ true;

23: FC_ReviseInitialDomain();

24: $\quad$ if ( $\forall v \in$ myInitialDomain, $\exists n g \in$ myNogoodStore $\mid R h s(n g)=v)$ then Backtrack ( ) ;

25: else CheckAssign (msg.Next)

procedure CheckAssign (next)

26: if $($ next $=$ self $)$ then Assign ();

procedure Backtrack ()

27: new Ng solve (myNogoodStore);

28: if $($ new $N g=$ empty $)$ then BroadcastMsg:Terminate $(\emptyset)$; end $\leftarrow$ true; return;

29: for each $x_{j} \succ$ Rhs $($ new $N g)$ do

30: myAgentView.Value $\left[x_{j}\right] \leftarrow$ unknown ;

31: for each $n g \in$ myNogoodStore do

32: $\quad$ if $\left(x_{j} \in \operatorname{Lhs}(n g)\right)$ then remove ( $n g$, myNogoodStore) ;

33: myAgentView.Consistent $\leftarrow$ false; myValue $\leftarrow$ empty; $C P A \leftarrow$ myAgentView;

34: SendMsg:BackCPA (CPA, new $N g)$ to Rhs $($ new $N g)$;

ProcessBackCPA $(m s g)$

35: if ( $\neg$ myAgentView.Consistent $\wedge$ myAgentView $\subset$ msg.CPA) then return;

36: splitlevel $\leftarrow$ CompareTimeStamp (myAgentView, msg.CPA);

37: if (splitlevel $=0 \wedge$ myValue $=$ RhsValue (msg.Nogood) $)$ then

38: add (msg.Nogood, myNogoodStore) ; myValue $\leftarrow$ empty; Assign() ;

ProcessTerminate $(\mathrm{msg})$

39: end $\leftarrow$ true; myValue $\leftarrow$ empty;

40: if (msg.CPA $\neq \emptyset)$ then myValue $\leftarrow$ msg.CPA.Value [self] ;

procedure UpdateMyAgentView ( $C P A$, splitlevel)

41: for each $j \geq$ splitlevel do myAgentView $[j] \leftarrow C P A[j]$; /* update value and Ctr */

42: for each $n g \in$ myNogoodStore do

43: if Lhs $(\mathrm{ng})$ is inconsistent with myAgentView then remove (ng, myNogoodStore) ;

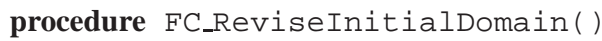

44: for each $v \in$ myInitialDomain do

45: $\quad$ if ( $\neg$ Consistent ( $v$, myAgentView) ) then

46: store the best nogood for $v ; / *$ according to the HPLV heuristic*/

function CompareTimeStamp (view, $C P A$ )

47: from $j \leftarrow 1$ to $\operatorname{size}(C P A)$ do

48: if $(C \operatorname{tr}(C P A[j])>C \operatorname{tr}(\operatorname{view}[j]))$ then return $j$;

49: else if $(C \operatorname{tr}(C P A[j])<C \operatorname{tr}($ view $[j]))$ then return -1 ;

50 : return 0 ;

Fig. 2. Nogood-based AFC algorithm running by agent self (Part 2) 
(lines 29-30). self remains in an inconsistent state until receiving a new CPA holding at least one agent assignment with counter higher than that in the agent view of self (lines 21-22).

When a $B a c k C P A$ message is received, sel $f$ checks the validity of received BackCPA using agent view status and time-stamp (lines 35-36). If $B a c k C P A$ is accepted (line 37), self removes its last assignment, adds attached nogood to its nogood store, and calls the procedure assign() (line 38).

ProcessTerminate procedure is called when an agent receives a Terminate message. It marks end flag true to stop the main loop (line 39). If attached CPA is empty then there is no solution. Otherwise, agent solution is retrieved from the CPA (line 40).

\section{Asynchronous Inter Level Forward-Checking}

A DisCSP can be represented by a constraint graph $G=(X, E)$, whose nodes represent the variables and edges represent the constraints (that is, $X=\mathcal{X}$ and $\left\{x_{i}, x_{j}\right\} \in E \Leftrightarrow$ $\left.c_{i j} \in \mathcal{C}\right)$. The graph can be re-arranged to form a pseudo-tree [14]. A pseudo-tree $G_{P T}=(X, r, E, U)$ for the graph $G$ is defined by a root node $r \in X$ and a directed tree $T=(X, U)$ rooted in $r$ such that for any edge $\left\{x_{i}, x_{j}\right\} \in E, x_{i}$ and $x_{j}$ are not in different branches of $T$. For any arc $\left(x_{i}, x_{j}\right) \in U$, the node $x_{i}$ is the parent of the node $x_{j}$. If $x_{i}$ is the parent of $x_{j}$, then $x_{j}$ is a child of $x_{i}$. A node $x_{i}$ is an ancestor of a node $x_{j}$ if $x_{i}$ is the parent of $x_{j}$ or an ancestor of the parent of $x_{j}$. A node $x_{j}$ is a descendant of a node $x_{i}$ if $x_{i}$ is an ancestor of $x_{j}$. A leaf is a node that has no child. In our implementation, the pseudo-tree is built by a DFS traversal of the graph. Thus, we have $U \subseteq E$.

The AILFC algorithm is based on AFC-ng performed on a pseudo-tree ordering of the constraint graph (built in a preprocessing step). Agents are prioritized according to the pseudo-tree ordering in which each agent has a single parent and various children. Using this priority ordering, AILFC performs multiple AFC-ng processes on the paths from the root to the leaves. The root initiates the search by generating a CPA, assigning its value on it, and sending CPA messages to its linked descendants (including its children) that share a constraint with it. Each child that receives a copy of the CPA performs AFC-ng on the sub-problem restricted to its ancestors (agents that are assigned in the CPA) and the set of its descendants. Therefore, instead of giving the privilege of assigning to only one agent, all agents who are in disjoint subtrees may assign their variables simultaneously. So, the Inter-Level Forward Checking is performed asynchronously on each path from the root to any leaf. AILFC thus exploits the potential speed-up of a parallel exploration in the processing of distributed problems.

An execution of AILFC on a sample DisCSP problem is shown in Figure 3. At time $t_{1}$, the root $x_{1}$ sends copies of the CPA on messages to its linked descendants (including its children). Children $x_{2}, x_{3}$ and $x_{4}$ assign their values simultaneously in the received CPAs and then perform concurrently the AILFC algorithm. Agents $x_{7}$, and $x_{9}$ only perform a forward- checking. At time $t_{2}, x_{9}$ finds an empty domain and sends a BackCPA message to $x_{1}$. At the same time, other CPAs propagate down through the other paths. For instance, a CPA has propagated down from $x_{3}$ to $x_{7}$ and $x_{8} . x_{7}$ detects an empty domain and sends a nogood to $x_{3}$ attached on a BackCPA message. 


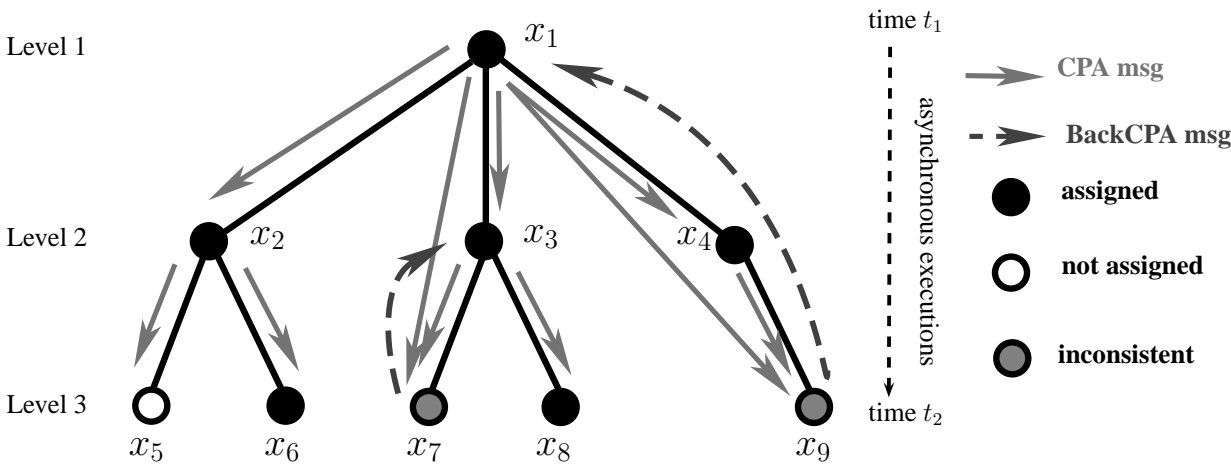

Fig. 3. An example of the AILFC execution

For the CPA that propagates on the path $\left(x_{1}, x_{2}, x_{5}\right)$ (resp. $\left.\left(x_{1}, x_{2}, x_{6}\right)\right), x_{5}$ (resp. $\left.x_{6}\right)$ successfully assigned its value and initiated a solution detection. However, when $x_{1}$ receives the $B a c k C P A$ from $x_{9}$, it initiates a new search process by sending a new copy of the CPA which will kill any CPA where $x_{1}$ is assigned its old value.

In AFC-ng, a solution is reached when the last agent receives the CPA and succeeds in assigning its variable. In AILFC, the situation is different because a CPA can reach a leaf without being complete. When all agents are assigned and no constraint is violated, this state is a global solution and the network has reached quiescence, meaning that no message is traveling through it. Such a state can be detected using specialized snapshot algorithms [15], but AILFC uses a different mechanism that allows to detect solutions before quiescence. AILFC uses an additional type of message called Accepted that inform parents of the acceptance of their CPA. Termination can be inferred earlier and the number of messages required for termination detection can be reduced. A similar technique of solution detection was used in the AAS algorithm [16].

The mechanism of solution detection is as follows: whenever a leaf node succeeds in assigning its value, it sends an Accepted message to its parent. This message contains the CPA that was received from the parent incremented by the value-assignment of the leaf node. When a non-leaf agent self receives Accepted messages from all its children that are all compatible with each other, all compatible with self's agent view and with self's value, self builds an Accepted message being the conjunction of all received Accepted messages plus self's value-assignment. If self is the root a solution is found, and self broadcasts this solution to all agents. Otherwise, self sends the built Accepted to its parent.

\section{Description of the algorithm}

A preprocessing step before starting the AILFC algorithm is performed to convert the constraint graph into a pseudo-tree. Children (self $) \subset \mathcal{A}$ is the set of children of agent self $f$ in the pseudo-tree, $\operatorname{Desc}(\operatorname{sel} f)$ is the set of its descendants and linkedDesc $(\operatorname{sel} f) \subset$ $\operatorname{Desc}(\operatorname{self})$ is the set of its descendants (including its children) that are constrained with self. Parent(self) $\in \mathcal{A}$ is the parent of agent self $f$ and Ancestors(self) $\subset \mathcal{A}$ is the set of its ancestors (including its parent).

In Figure 4, we present only the procedures that are new or different from those of AFC-ng in Figures 1 and 2. In InitMyAgentView(), the agent view of self $f$ is initialized 


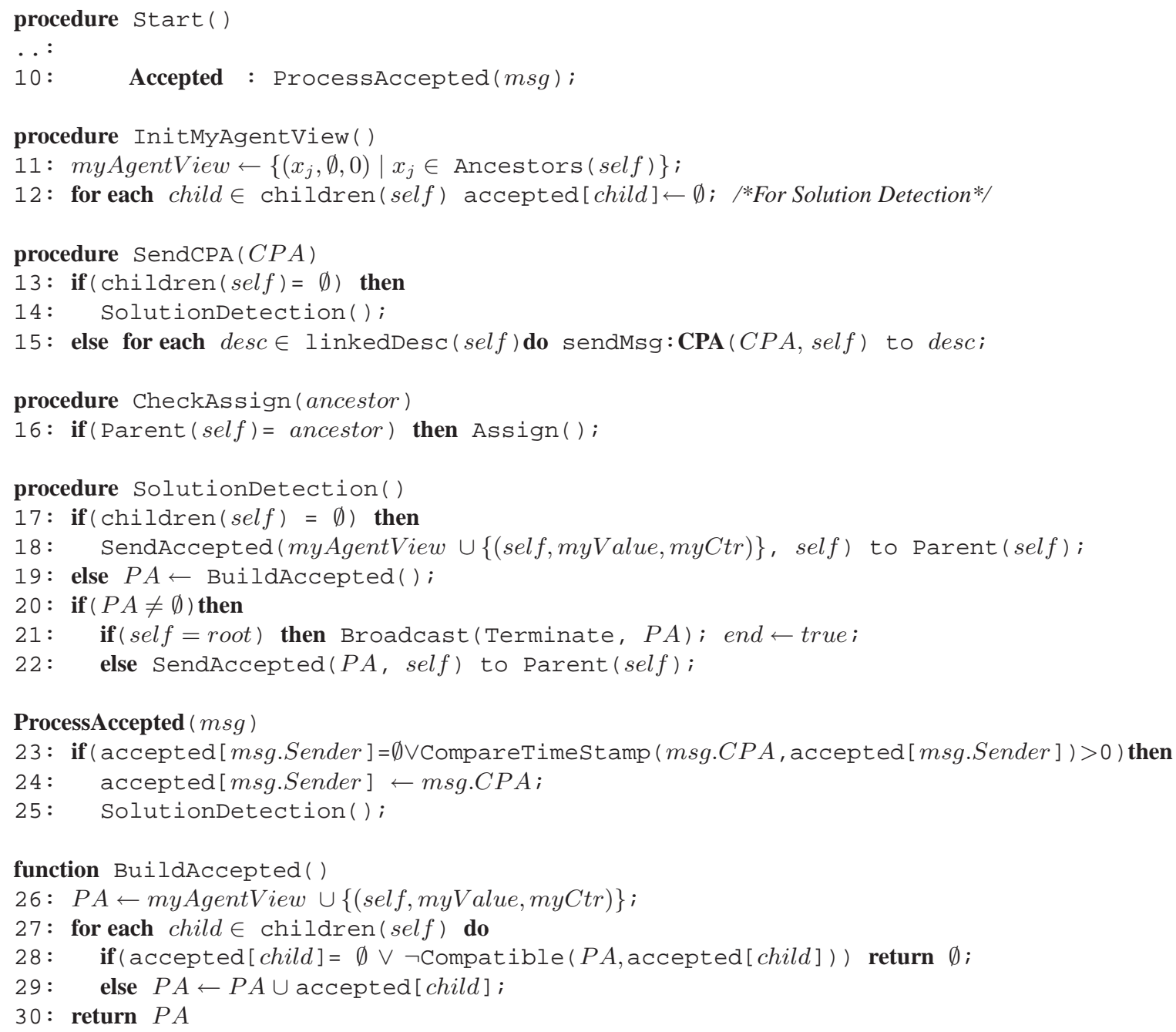

Fig. 4. New lines/procedures of AILFC with respect to AFC-ng.

to the set Ancestors(self). Ctr is set to 0 for each agent in Ancestors(self) (line 11). The new data structure storing the received Accepted messages is initialized to the empty set (line 12). In SendCPA $(C P A)$, instead of sending copies of the CPA to all agents not yet instantiated on it, self sends copies of the CPA only to its linked descendants (linkedDesc(self)) (line 15). When the set linkedDesc(self) is empty (i.e., self is a leaf), self calls the procedure SolutionDetection to build and send an Accepted message. In CheckAssign(ancestor), self assigns its value if the CPA was received from its parent (line 16) (i.e., if ancestor is the parent of self).

In SolutionDetection(), if self is a leaf (Children(self) is empty), it sends an Accepted message to its parent. The Accepted message sent by self $f$ contains its agent view incremented by its assignment (lines 17-18). If self is not a leaf, it calls the BuildAccepted() procedure to build an accepted partial solution $P A$ (line 19). If the 
returned partial solution $P A$ is not empty and sel $f$ is the root, $P A$ is a solution of the problem. Then, self broadcasts it to other agents including the system agent and sets the end flag to true (line 21). Otherwise, self sends an Accepted message containing $P A$ to its parent (line 22).

In ProcessAccepted $(\mathrm{msg})$, when sel $f$ receives an Accepted message from its child for the first time, or when $m s g$ is newer than that received before (lines 23-24), self stores the content of this message and calls the SolutionDetection procedure (line 25).

In BuildAccepted(), if an accepted partial solution is reached. self generates a partial solution $P A$ incrementing its agent view with its assignment (line 26). Next, self loops over the set of Accepted messages received from its children. If at least one child has never sent an Accepted message or the Accepted message is incompatible with $P A$, then the partial solution has not yet been reached and the function returns empty (lines 27-28). Otherwise, the partial solution $P A$ is incremented by the Accepted message of child (line 29). Finally, the accepted partial solution is returned (line 30).

\section{Correctness Proofs}

Theorem 1. AFC-ng is sound, complete, and terminates.

The argument for soundness is close to the one given in [11,17]. The fact that agents only forward consistent partial solution on the CPAs messages at only one place in function assign() (line 14), implies that the agents receive only consistent assignments. A solution is reported by the last agent only in function $\operatorname{SendCPA}(C P A)$ at line 17. At this point, all agents have assigned their variables, and their assignments are consistent. Thus the AFC-ng algorithm is sound.

For completeness, we need to show that AFC-ng is able to terminate and does not report inconsistency if a solution exists.

Lemma 1. AFC-ng is guaranteed to terminate.

For sake of clarity, we assume that the order in which AFC-ng assigns the variables is the lexicographic ordering $X_{1}, X_{2}, \ldots, X_{n}$. We define the total order $o$ on CPAs as follows. Let $I_{1}$ be an assignment on $X_{1}, \ldots, X_{k_{1}}, I_{2}$ be an assignment on $X_{1}, \ldots, X_{k_{2}}$, and $s$ be the smallest index on which $I_{1}$ and $I_{2}$ differ. $I_{1} \prec{ }_{0} I_{2}$ if and only if $s=k_{1}+1$ or the value $I_{1}[s]$ is chosen before the value $I_{2}[s]$ by the value ordering heuristics on variable $X_{s}$ given the CPA $I_{1}[1 . . s-1]$.

To prove the lemma we prove that AFC-ng performs a finite number of backtrack steps. In AFC-ng, several backtracks can be performed simultaneously as they are generated concurrently by different agents to different destinations. The re-assignments of destination agents then happen simultaneously, generating several CPAs. However, the CPA at the highest level in the search hierarchy tree will eventually dominate all others thanks to its greater time-stamp (see line 21 in Figure 2). Thus, every backtrack step may be represented by the backtrack at the highest level. The agent $X_{i}$ who has received that backtrack of highest level has to replace its previous assignment $v_{i}$ in the CPA by a new one $v_{i}^{\prime}$ because the backtrack message contains a nogood rejecting value $v_{i}$. If $v_{i}$ was not the first value chosen by $X_{i}$ since it has received the current CPA from 
$X_{i-1}$ then we know that all other values $v_{j}$ preferred to $v_{i}$ were ruled out by a nogood at the time $v_{i}$ was chosen. Now, the CPA on $X_{1}, \ldots, X_{i-1}$ has not changed since then, otherwise this would not be the highest backtrack. As a result, the nogoods rejecting values $v_{j}$ preferred to $v_{i}$ are still valid and $v_{i}^{\prime}$ is necessarily the next preferred value in the heuristic order. By definition of the order $o$, the new CPA obtained is greater than the previous one according to $o$ because it has not changed on $X_{1}, \ldots, X_{i-1}$ and $v_{i}^{\prime}$ is less preferred than $v_{i}$. Since $o$ is a total order and since there are a finite number of variables and a finite number of values per variable, there will be a finite number of new CPAs generated. Now, each backtrack of highest level generates a new CPA. Thus, AFC-ng performs a finite number of backtracks.

Lemma 2. AFC-ng cannot infer inconsistency if a solution exists.

Whenever a newer CPA or a $B a c k C P A$ message is received, AFC-ng agent updates its nogood store. Hence, for every CPA that may potentially lead to a solution, agents only store valid nogoods. In addition, every nogood resulting from a CPA is redundant with regard to the DisCSP to solve. Since all additional nogoods are generated by logical inference when a domain wipe-out occurs, the empty nogood cannot be inferred if the network is satisfiable. This mean that AFC-ng is able to produce all solutions.

Theorem 2. AILFC algorithm is sound, complete, and terminates.

AILFC agents only forward consistent partial assignments (CPAs). Hence, leaf agents receive only consistent CPAs. Thus, leaf agents send Accepted message only holding consistent assignments to their parent. Since a parent builds an Accepted message only when the Accepted messages received from all its children are compatible with each other and all compatible with its own value, the Accepted message it sends contains a consistent partial solution. The root broadcasts a solution only when it can build itself such an Accepted message. Therefore, the solution is correct and AILFC is sound.

AILFC performs multiple AFC-ng processes on the paths of the pseudo-tree from the root to the leaves. Thus, it inherits the completeness property of AFC-ng (empty nogood cannot be inferred if the network is satisfiable (see Lemma 2). It also appears that the agent of high priority cannot fall into an infinite loop. By induction on the level of the pseudo-tree no agent can fall in such a loop, which ensures the termination of AILFC.

\section{Experimental Evaluation}

In this section we compare experimentally AFC-ng and AILFC to three other algorithms: AFC, ABT, and ABT-Hyb [18]. Algorithms are tested on the same static agents ordering using max-degree heuristic and the same nogood selection heuristic (HPLV). For ABT and ABT-Hyb we implemented an improved version of Silaghi's solution detection [12] and counters for tagging assignments. This allows to better treat non-causal order channels [12]. All experiments were performed on the DisChoco platform [19] in which agents are simulated by Java threads that communicate only through message passing. 


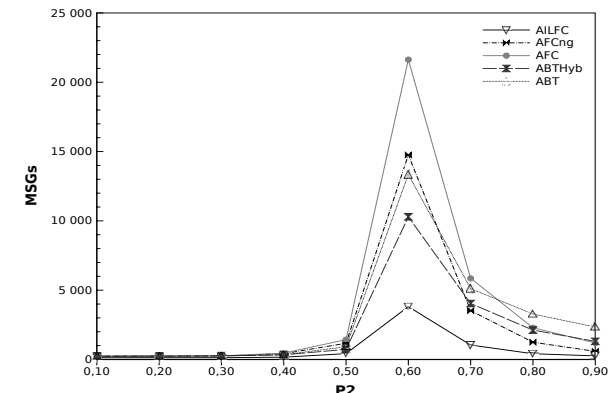

(a) $\left\langle n=20, d=10, p_{1}=0.25\right\rangle$

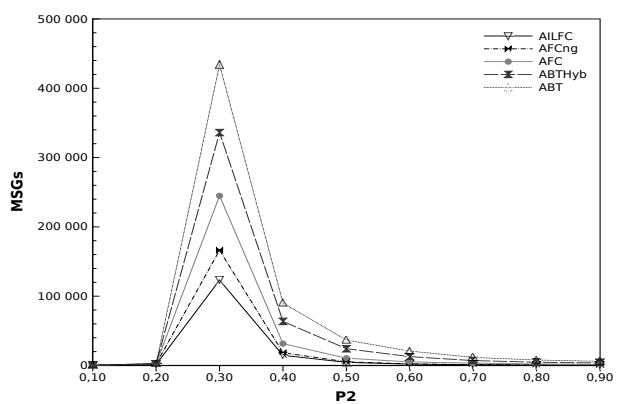

(c) $\left\langle n=20, d=10, p_{1}=0.75\right\rangle$

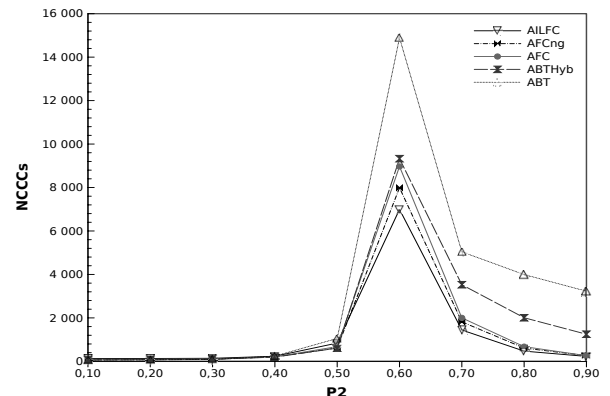

(b) $\left\langle n=20, d=10, p_{1}=0.25\right\rangle$

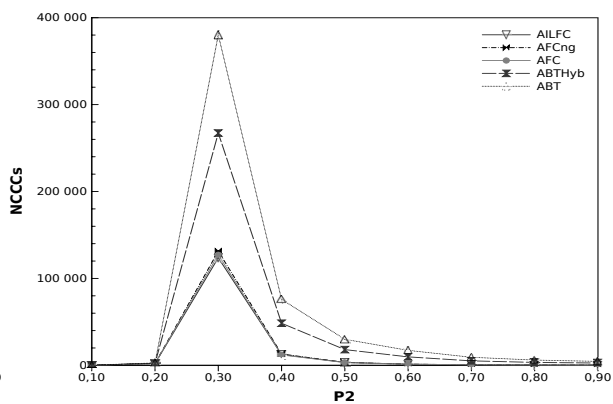

(d) $\left\langle n=20, d=10, p_{1}=0.75\right\rangle$

Fig. 5. Total number of messages sent and NCCCs on fast communication

The algorithms are tested on uniform binary random DisCSPs which are characterized by $\left\langle n, d, p_{1}, p_{2}\right\rangle$, where $n$ is the number of agents/variables, $d$ the number of values per variable, $p_{1}$ the network connectivity defined as the ratio of existing binary constraints, and $p_{2}$ the constraint tightness defined as the ratio of forbidden value pairs. We solved 100 instances of two classes of constraints graph: sparse graph $\left\langle 20,10,0.25, p_{2}\right\rangle$ and dense graph $\left\langle 20,10,0.75, p_{2}\right\rangle$. We vary the tightness from 0.10 to 0.90 by steps of 0.10 .

We evaluate the algorithms performance by the average of total messages sent [20] (including system messages) and the average of Equivalent Non-Concurrent Constraint Checks (ENCCCs) [21]. ENCCCs are a weighted sum of processing and communication time. We simulate two scenarios of communication: fast communication (where message delay is null and ENCCCs reduce to standard NCCCs), and slow communication with uniform random message delay (where the cost of the delay is between 500 and 1000 constraint checks.)

\section{Fast communication}

Figure 5 presents performance of AILFC, AFC-ng, AFC, ABT and ABT-hyb running on a fast communication environment. The figure shows that in both types of constraint graphs (sparse and dense), AILFC has the lowest communication load (\#MSGs). Concerning NCCCs, AILFC is the fastest algorithm on sparse graphs (Fig. 5(b)). On dense 


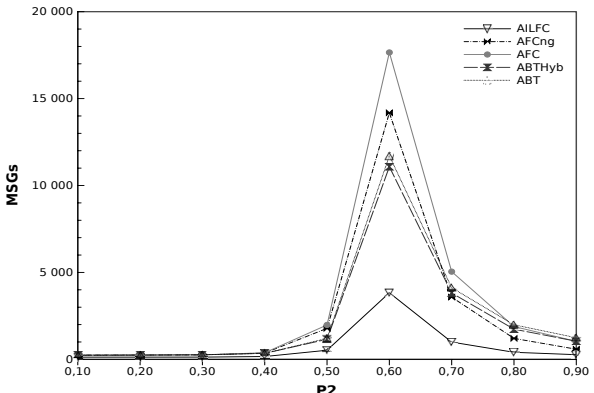

(a) $\left\langle n=20, d=10, p_{1}=0.25\right\rangle$

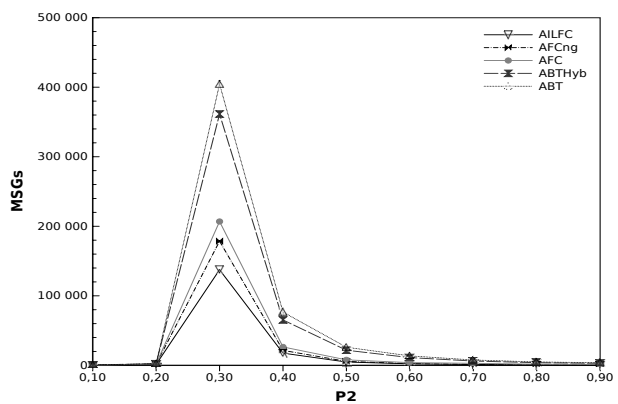

(c) $\left\langle n=20, d=10, p_{1}=0.75\right\rangle$

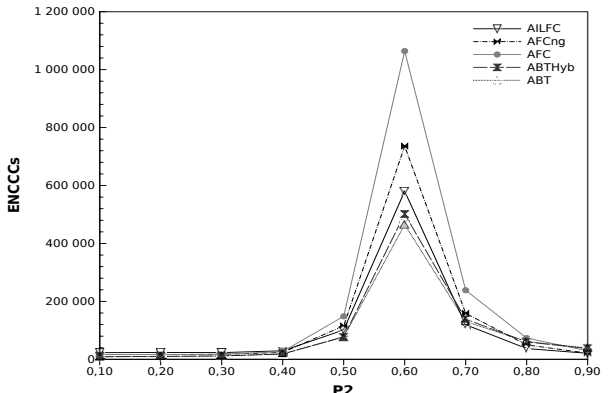

(b) $\left\langle n=20, d=10, p_{1}=0.25\right\rangle$

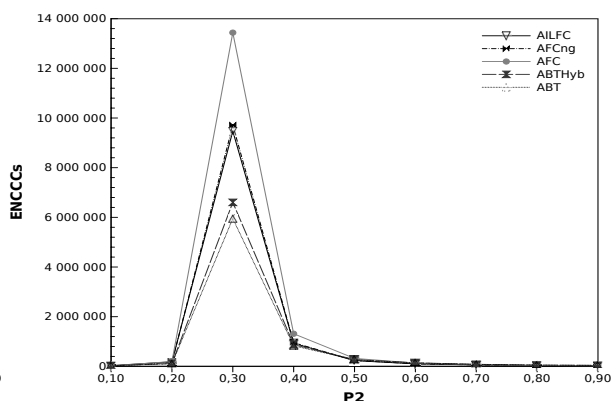

(d) $\left\langle n=20, d=10, p_{1}=0.75\right\rangle$

Fig. 6. Total number of messages sent and ENCCCs on slow communication

graphs AILFC behaves like AFC and AFC-ng. Comparing AFC-ng with AFC, Fig. 5 shows that they perform the same number of NCCCs but AFC-ng exchanges less messages than AFC. Comparing AFC-ng with ABT and ABT-hyb, Fig. 5 shows that in both types of constraint graphs AFC-ng is faster than ABT-hyb and ABT. However, on sparse graphs, Fig 5(a) shows that AFC-ng sends more messages than ABT-hyb and ABT.

\section{Slow communication}

In Figure 6 we report experimental results with slow communication. The figure shows that AILFC is again the best algorithm in terms of number of messages. Concerning ENCCCs, as in the fast communication environment, AILFC is faster than or equal to $\mathrm{AFC}$ and AFC-ng depending on whether the graph is sparse or dense. The comparison of AFC and AFC-ng shows a pattern close to the one observed with fast communication: AFC-ng is better, or slightly better, both in terms of messages and ENCCCs. The main difference between fast and slow communication is the performance of ABT and ABT-hyb. Whereas they remain expensive in terms of messages, they become the best algorithms in terms of ENCCCs, with a slight advantage to ABT. This confirms that in slow communication environment, the more the algorithm is asynchronous, the better it is. 


\section{Discussion}

A first observation on these experiments is that ABT, ABT-hyb on one side, and AFC, AFC-ng on the other side, show quite opposite patterns. If message passing is not an issue, ABT and ABT-hyb are good choices with slow communication whereas AFC and AFC-ng are good when communication is fast. A second observation is that AILFC is always better than or equivalent to AFC-ng, which is better than or equivalent to AFC, both in terms of messages and amount of processing (ENCCCs). If limiting the communication load is important, AILFC is the best among all both for fast and slow communication. AILFC benefits both from running separate search processes in disjoint problem subtrees, which pays off when a graph is sparse, and from using the same mechanism as AFC-ng, which pays off when agents are highly connected (dense graphs).

\section{Other Related Work}

In $[18,7]$ the performance of asynchronous (ABT), synchronous ( Synchronous Conflict BackJumping (SCBJ)), and hybrid approaches (ABT-Hyb) was studied. It is shown that $\mathrm{ABT}-\mathrm{Hyb}$ improves over $\mathrm{ABT}$ and that $\mathrm{SCBJ}$ requires less communication effort than ABT-Hyb. In Interleaved Asynchronous Backtracking (IDIBT) [22], agents participate in multiple processes of asynchronous backtracking. Each agent keeps a separate AgentView for each search process in IDIBT. The number of search processes is fixed by the first agent in the ordering. The performance of concurrent asynchronous backtracking [22] was tested and found to be ineffective for more than two concurrent search processes [22]. Dynamic Distributed BackJumping (DDBJ) was presented in [17]. It is an improved version of the basic AFC. It combines the concurrency of an asynchronous dynamic backjumping algorithm, and the computational efficiency of the AFC algorithm, coupled with the possible conflict heuristics of dynamic value and variable ordering. As in DDBJ, AFC-ng performs several backtracks simultaneously. However, AFC-ng should not be confused with DDBJ. DDBJ is based on dynamic ordering and requires additional messages to compute ordering heuristics. In AFC-ng, all agents that received a $B a c k C P A$ message continue search concurrently. Once a more up to date CPA is received by an agent, all nogoods already stored can be kept if consistent with that CPA.

\section{Conclusion}

Two new complete, asynchronous algorithms are presented. The first algorithm, NogoodBased Asynchronous Forward Checking (AFC-ng), is an improvement on AFC. The second, Asynchronous Inter-Level Forward-Checking (AILFC), is based on AFC-ng and is performed on a pseudo-tree re-arrangement of the constraint graph. Experiments ran on random DisCSPs show that AFC-ng improves AFC both in fast and slow communication environments. Experiments show that AILFC is the more robust algorithm in both communication types. In particular, it is the best in terms of messages sent. In slow communication environments, the performance of algorithms that perform variable assignments sequentially deteriorates. This is observed for AFC and AFC-ng, and, less significantly for ABT-Hyb, when compared to ABT. 


\section{References}

1. Petcu, A., Faltings, B.: A value ordering heuristic for distributed resource allocation. In: Proceeding of CSCLP04, Lausanne, Switzerland (2004)

2. Wallace, R.J., Freuder, E.: Constraint-based multi-agent meeting scheduling: effects of agent heterogeneity on performance and privacy loss. In: Proceeding of the 3rd workshop on distributed constrait reasoning, DCR-02, Bologna (2002) 176-182

3. Fernandez, C., Bejar, R., Krishnamachari, B., Gomes, K.: Communication and computation in distributed CSP algorithms. In: Proceeding of CP-02, Ithaca, NY, USA (2002) 664-679

4. Chong, Y.L., Hamadi, Y.: Distributed log-based reconciliation. In: ECAI. (2006) 108-112

5. Yokoo, M.: Algorithms for distributed constraint satisfaction problems: A review. Autonomous Agents \& Multi-Agent Systems 3 (2000) 198-212

6. Yokoo, M., Durfee, E.H., Ishida, T., Kuwabara, K.: Distributed constraint satisfaction problem: Formalization and algorithms. IEEE Trans. on Data and Kn. Eng. 10 (1998) 673-685

7. Zivan, R., Meisels, A.: Synchronous vs asynchronous search on DisCSPs. In: Proceeding of 1st European Workshop on Multi Agent System, EUMAS, Oxford (2003)

8. Yokoo, M., Durfee, E.H., Ishida, T., , Kuwabara., K.: Distributed constraint satisfaction for formalizing distributed problem solving. In: IEEE Intern. Conf. Distrb. Comp. Sys. (1992) 614-621

9. Bessiere, C., Maestre, A., Brito, I., Meseguer, P.: Asynchronous backtracking without adding links: a new member in the ABT family. Artificial Intelligence 161:1-2 (2005) 7-24

10. Meisels, A., Zivan, R.: Asynchronous forward-checking for distributed CSPs. In Zhang, W., ed.: Frontiers in Artificial Intelligence and Applications, IOS Press (2003)

11. Meisels, A., Zivan, R.: Asynchronous forward-checking for DisCSPs. Constraints 12(1) (2007) 131-150

12. Silaghi, M.C.: Generalized dynamic ordering for asynchronous backtracking on DisCSPs. In: DCR workshop, AAMAS-06, Hakodate, Japan (2006)

13. Maestre, A., Bessiere, C.: Improving asynchronous backtracking for dealing with complex local problems. In: ECAI. (2004) 206-210

14. Freuder, E.C., Quinn, M.J.: Taking advantage of stable sets of variables in constraint satisfaction problems. In: In IJCAI85. (1985) 1076-1078

15. Mani, K.C., Lamport, L.: Distributed snapshots: determining global states of distributed systems. ACM Trans. Comput. Syst. 3(1) (1985) 63-75

16. Silaghi, M.C., Faltings, B.: Asynchronous aggregation and consistency in distributed constraint satisfaction. Artificial Intelligence 161:1-2 (2005) 25-54

17. Nguyen, T., Sam-Hroud, D., Faltings, B.: Dynamic distributed backjumping. In: Proceeding of 5th workshop on DCR-04, Toronto (2004)

18. Brito, I., Meseguer, P.: Synchronous, asynchronous and hybrid algorithms for DisCSP. In: Workshop on DCR-04, CP-2004, Toronto (2004)

19. Ezzahir, R., Bessiere, C., Belaissaoui, M., Bouyakhf, E.H.: Dischoco: a platform for distributed constraint programming. In: Proceeding of Workshop on Distributed Constraint Reasoning of IJCAI-07. (2007) 16-21

20. Lynch, N.A.: Distributed Algorithms. Morgan Kaufmann Series (1997)

21. Chechetka, A., Sycara, K.: No-commitment branch and bound search for distributed constraint optimization. In: Proc. of AAMAS '06, New York, NY, USA, ACM (2006) 14271429

22. Hamadi, Y.: Interleaved backtracking in distributed constraint networks. Int.. J. of AI Tools 11 (2002) 167-188 